\title{
SOCIAL HETEROGENEITY AND THE COVID-19 LOCKDOWN IN A MULTI-GROUP SEIR MODEL
}

\author{
JEAN DOLBEAULT ${ }^{(1)}$ AND GABRIEL TURINICI ${ }^{(2)}$ \\ CEREMADE (CNRS UMR n 7534), PSL University, Université Paris-Dauphine, \\ Place de Lattre de Tassigny, 75775 Paris 16, France \\ ${ }^{(1)}$ E-mail: dolbeaul@ceremade.dauphine.fr, ${ }^{(2)}$ E-mail: turinici@ceremade.dauphine.fr
}

\begin{abstract}
The goal of the lockdown is to mitigate and if possible prevent the spread of an epidemic. It consists in reducing social interactions. This is taken into account by the introduction of a factor of reduction of social interactions $q$, and by decreasing the transmission coefficient of the disease accordingly. Evaluating $q$ is a difficult question and one can ask if it makes sense to compute an average coefficient $q$ for a given population, in order to make predictions on the basic reproduction rate $\mathcal{R}_{0}$, the dynamics of the epidemic or the fraction of the population that will have been infected by the end of the epidemic. On a very simple example, we show that the computation of $\mathcal{R}_{0}$ in a heterogeneous population is not reduced to the computation of an average $q$ but rather to the direct computation of an average coefficient $\mathcal{R}_{0}$. Even more interesting is the fact that, in a range of data compatible with the Covid-19 outbreak, the size of the epidemic is deeply modified by social heterogeneity, as is the height of the epidemic peak, while the date at which it is reached mainly depends on the average $\mathcal{R}_{0}$ coefficient. This paper illustrates more technical results that can be found in [4], with new numerical computations. It is intended to draw the attention on the role of heterogeneities in a population in a very simple case, which might be difficult to apprehend in more realistic but also more complex models.
\end{abstract}

The model. We consider a compartmental model based on the SEIR equations (for Susceptible, Exposed, Infected, Recovered) with $n$ categories of susceptible individuals and a total number of individuals $N$ given by

$$
\frac{d S_{k}}{d t}=-\beta_{k} S_{k} \frac{I}{N}, \quad \frac{d E}{d t}=\sum_{k=1}^{n} \beta_{k} S_{k} \frac{I}{N}-\alpha E, \quad \frac{d I}{d t}=\alpha E-\gamma I, \quad \frac{d R}{d t}=\gamma I .
$$

We consider this system as a function of $t \geq 0$ in terms of the initial data corresponding to the values of $S, E, I$ and $R$ at $t=0$. The average incubation period is $1 / \alpha$, the parameter $\beta_{k}$ is the product of the average number of contacts per person and per unit time by the probability of disease transmission in a contact between a susceptible individual in the group $k$ and any infectious individual, $\gamma$ is a transition rate so that $1 / \gamma$ measures the duration of the infection of an individual (or actually how long he is infectious and able to contaminate other people before being isolated), and $N$ is the total population size.

An individual in the group $k$ is characterized by his transmission rate $\beta_{k}$. In simple SEIR models of lockdown, a single group is considered $(n=1)$ and it has been proposed for instance in [7] to introduce a factor of reduction of social

2020 Mathematics Subject Classification. Primary: 92C60; Secondary: 92D30, 34C60.

Keywords: Epidemic models; Disease control; Heterogeneous populations; Basic reproduction ratio; Equilibrium solutions. 
interactions $q$ so that the effect of the lockdown is to reduce the transmission rate from $\beta_{1}$ before lockdown to $\beta_{1} / q$ after lockdown. Our goal is to study what happens if there are, after lockdown, $n \geq 2$ groups with different factors of reduction of social interactions $q_{k}$, so that

$$
\beta_{k}=\frac{\beta}{q_{k}},
$$

where $\beta$ is a fixed, given parameter. We shall speak of social groups and social heterogeneity because each group has its own transmission rate $\beta_{k}$. This rate does not interfere with the dynamics of the disease once the corresponding individual is infected. In a period of lockdown, the reduction factor is not the same for a health worker, a supermarket cashier or an employee working from home by internet. Actually $\beta_{k}$ can also be used to take into account other characteristics of the population, like age groups, which play a role in the risk of becoming infected. Here we assume that $q_{k}$ determines the initial group of an individual and does not vary over time. The other coefficients $\alpha, \beta$ and $\gamma$ of System (1) are also assumed to be independent of $t$. A last point of terminology concerns the notion of individuals. As we use a compartmental model, we should speak only of the proportion of individuals in a compartment. Since the total number of individuals $N$ is taken large, this proportion can be considered as a continuous variable subject to ordinary differential equations, which is precisely what we do in (1).

Reduction of social interactions and basic reproduction ratio. Let us consider the initial probability distribution among the groups such that, at $t=0$,

$$
S_{k}(0)=p_{k} S(0) \quad \text { where } \quad S(0)=\sum_{k=1}^{n} S_{k}(0) .
$$

Notice that $p_{k}$ is a parameter used for the description of the initial datum only. In a disease free equilibrium corresponding to $S=N$, it would be very natural to consider an average factor of reduction of social interactions

$$
q=\sum_{k=1}^{n} p_{k} q_{k}
$$

and this is actually what is done implicitly when a single compartment of susceptible individuals is considered. In that case, the basic reproduction ratio computed for instance by the next generation matrix method (see [3] and references therein) is $\beta /(q \gamma)$. However, if we apply the next generation matrix method to (1), we found in $[4$, Theorem $4.2(1)]$ that the basic reproduction ratio is

$$
\mathcal{R}_{0}=\sum_{k=1}^{n} p_{k} \frac{\beta_{k}}{\gamma} .
$$

Although this is a relatively elementary property, there are already important consequences, for instance in the case $\frac{\beta}{q \gamma}<1<\mathcal{R}_{0}$. While a model with a single compartment and an average factor of reduction of social interactions predicts the extinction of the epidemic, a small group with high transmission factor $\beta_{k}$, even if numerically not very large (that is, with $p_{k}$ small), can spread the disease and trigger an outbreak. This is one of the possible interpretations of the data, which were suggesting a pattern corresponding to a basic reproduction ratio larger than 1 in the initial stage of the lockdown in France rather than an expected exponential decay of the number of cases. 
Limitations and choice of the numerical parameters. System (1) is an extremely simplified model, in which Undetected or asymptomatic cases are not taken into account, although this seems an important issue in the Covid-19 pandemic. It is also a model for short term (say of the order of three months) so that the evolution of the structure as well as natural birth and death rates are not taken into account. If Covid-19 becomes endemic or last longer, it will not be possible to keep ignoring such issues anymore. Feedback mechanisms and changes in the values of the parameters due to the evolution of the disease or the changes in social habits are certainly going to play a role. More important is the fact that the parameters are fitted on the basis of official data during the very early stage of the disease.

System (1) is homogeneous so that we can simply consider the fractions

$$
\mathrm{s}_{k}:=\frac{S_{k}}{N}, \quad \mathrm{~s}:=\frac{S}{N}, \quad \mathrm{e}:=\frac{E}{N}, \quad \mathrm{i}:=\frac{I}{N}, \quad \mathrm{r}:=\frac{R}{N}
$$

of the Susceptible, Exposed, Infected and Recovered individuals among the whole population as a function of the time $t \geq 0$. Numerical values are taken from [2]: for the initial data, at $t=0$, we consider a perturbation of the disease free equilibrium given by $\mathrm{s}_{k}(0)=p_{k} \mathbf{s}(0)$ and

$$
\mathrm{s}(0)=0.99981, \quad \mathrm{e}(0)=8.81 \times 10^{-5}, \quad \mathrm{i}(0)=1.88 \times 10^{-5}, \quad \mathrm{r}(0)=8.04 \times 10^{-5},
$$

based on the data of March 15, 2020 in France from [8], and choose in (1)

$$
\beta=2.33, \quad \alpha=0.25, \quad \gamma=1,
$$

which gives us a basic reproduction ratio of 2.33 (in the case of a single group). The reader interested in further details and comparisons is invited to refer to [4], where the choice of the numerical parameters is discussed in greater details. This set of initial data and parameters is however not a key issue for our discussion. Our contribution is focused on understanding the theoretical implications of social heterogeneity and remains valid for other sets of numerical choices. Numerical choices (3)-(4) are given only for an illustrative purpose.

In [2], N. Bacaër is able to fit the curve of the number of infected individuals with a single group and $q_{1}=1.7$, at the beginning of the lockdown. In [4], it is shown that with two groups, $p_{1}=98 \%, p_{2}=2 \%, q_{1}=2.35$ and $q_{2}=0.117$, we can recover the same reproduction ratio of 1.37 as in [2]. In order to fix ideas, we take

$$
q_{1}=2.4
$$

which corresponds to a reduction of the transmission rate of $1 / q_{1} \approx 42 \%$ in the group $k=1$. We emphasize that we have no empirical basis to determine this parameter. The idea is to pick for $q_{1}$ a value larger than 2.33 so that, in presence of a single group, the epidemic would rapidly extinguish. However, a second group with a moderate or high transmission coefficient $\beta_{2}$ is enough to produce an outbreak corresponding to a global $\mathcal{R}_{0}>1$ and we study how various features of the epidemic curve depend on $\beta_{2}$. See Fig. 1 .

From now on, we shall assume in all our numerical results that $n=2$ (two groups) and vary $p=p_{2}$ (so that $p_{1}=1-p$ ). Consistently with the idea that the initial stage of the outbreak is independent of the groups, we take as initial data $\mathrm{s}_{1}(0)=(1-p) \mathrm{s}(0)$ and $\mathrm{s}_{2}(0)=p \mathrm{~s}(0)$. We keep $p$ small and take it in the range 1 to $5 \%$ in our examples. It is not difficult to understand that the destabilization due to a small fraction $p$ of the population (the $k=2$ group) in the large group (the $k=1$ 


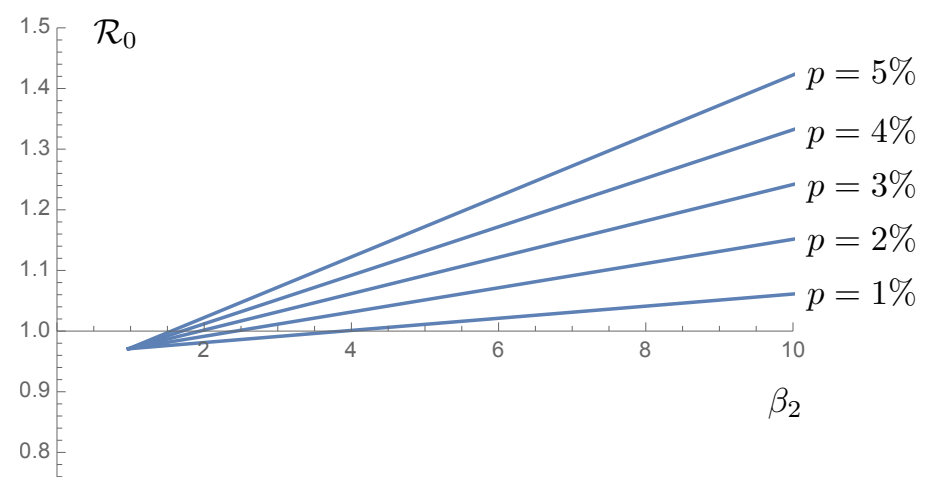

FiguRE 1. Numerical values of $\mathcal{R}_{0}$ : we take $p$ small but assume that the individuals in the group $k=2$ may have a moderate or high transmission coefficient $\beta_{2}$.

group) in which the epidemic would be under control if it were isolated, is all the stronger when the basic reproduction ratio (inside the $k=1$ group, considered as isolated) is close to 1 : this is the reason why we arbitrarily choose $q_{1}=2.4$. The case of $q_{2}$ small (eventually smaller than 1), corresponding to individuals in the group $k=2$ with high transmission rates, is of particular interest.

Our model is overly simplified. More realistic models should describe social interactions with far more details, address not only lockdown but also mask wearing and disinfection measures, social-distancing, self-quarantine, various types of stayat-home orders and curfews, etc., and also take into account the evolution of the parameters induced by behavioural changes and economical constraints as studied for instance in [6].

The epidemic size. The (final) epidemic size $\zeta$ is defined as the fraction of individuals that are affected by the epidemic for large times, here $s(0)-s^{\star}$, where $s^{\star}$ is the fraction which is not infected at the end of the epidemic. Under the condition that $\mathcal{R}_{0}>1$ and $1-\mathrm{s}(0)$ is small, we find that $\mathrm{r}^{\star}=1-\mathrm{s}^{\star}$ is of the order of the solution $r$ of

$$
\sum_{k=1}^{n} e^{-\frac{\beta_{k} r}{\gamma}}+r=1
$$

(see in [4, Section 3.2 and Theorem $4.2(1)]$ ). It is also proved in [4, Theorem $4.2(3)]$ that $\zeta$ is decreased when $n$ is increased from $n=1$ to an arbitrary $n \geq 2$ (for our numerical examples, we take $n=2$ ). See Figs. 2 and 3. In Fig. $3, \mathcal{R}_{0}=1.37$ is achieved in a single group with $q \approx 1.70$ as in [2], which gives an epidemic size $\zeta \approx 49 \%$. The same value $\mathcal{R}_{0}=1.37$, which is obtained in two groups with $p=1 \%$, $q_{1}=2.4$ (and $q_{2} \approx 0.06$ ), gives an epidemic size $\zeta \approx 12 \%$. 


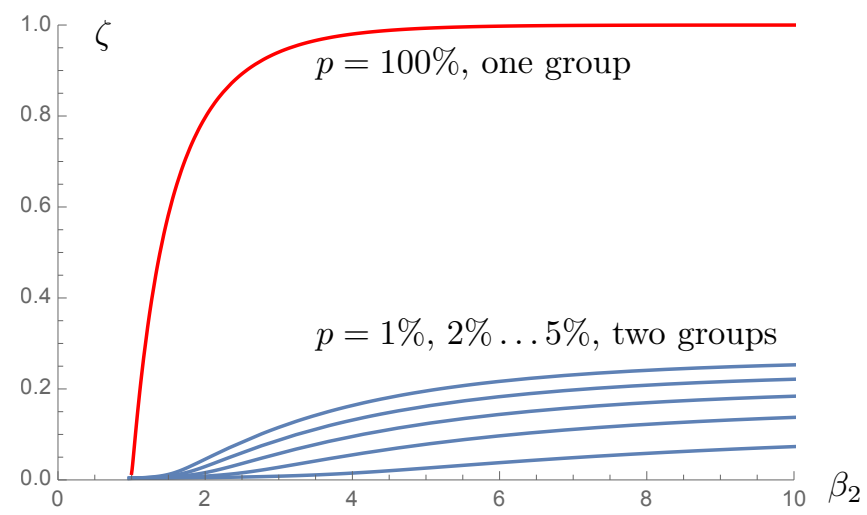

Figure 2. Epidemic size $\zeta$ as a function of $\beta_{2}$ for $p=0.01$, $0.02, \ldots 0.05$ (blue; $n=2$ ) and $p=1$ (red, $n=1$ ). This red curve corresponds to an outbreak with a basic reproduction ratio $\beta_{2} / \gamma$. This is however not so instructive as the $k=2$ group is a small proportion of the population, but with high transmission rate.

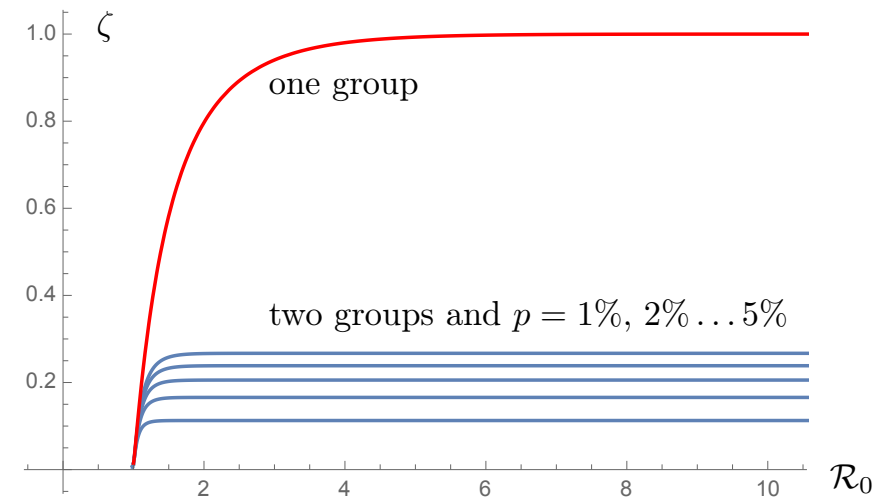

Figure 3. Epidemic size $\zeta$ as a function of $\mathcal{R}_{0}$ for $p=0.01$, $0.02, \ldots 0.05$ (blue) and $p=1$ (red), which corresponds to the case of a single group. With the same $\mathcal{R}_{0}=1.37$, we see that $\zeta$ is drastically decreased.

The epidemic peak. Not only the asymptotics of the epidemic are changed when replacing the model with $n=1$ by a model with $n=2$, but also the dynamical behavior of the solutions. The height of the epidemic peak (defined as the maximum of infected $\mathrm{e}+\mathrm{i}$ as function of time) is decreased when $n$ is increased form $n=1$ to $n \geq 2$ : see [4] for a proof. The height and date of the epidemic peak strongly depend on $p$. See Figs. 4, 5 and 6 . In Fig. 5 , for the same value of $\mathcal{R}_{0} \approx 1.5$, the curve $t \mapsto \mathrm{e}(t)+\mathrm{i}(t)$ is represented in red for a single group (this corresponds here to a factor of reduction of social interactions $q \approx 1.55$ ) and in blue in the case of two groups (with parameters $q_{1}=2.4, q_{2}=0.2$ and $p=0.05$ ). 


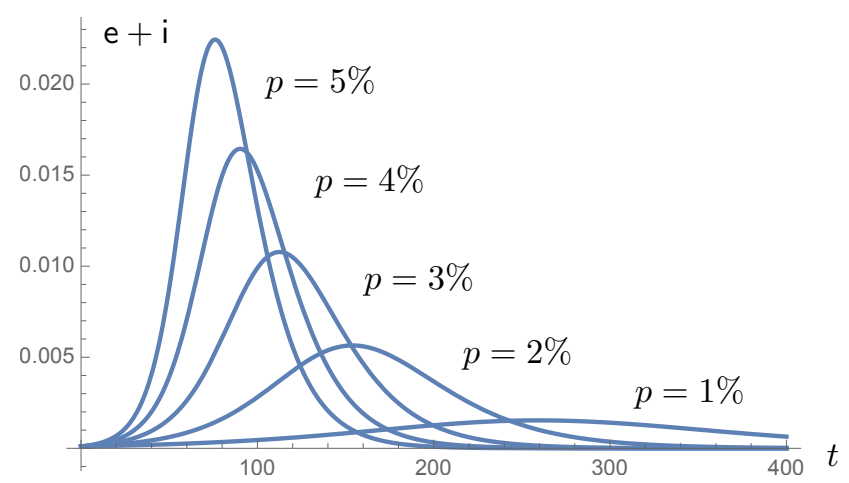

Figure 4. Fraction of exposed and infected individuals e $+\mathrm{i}$ as a function of time (in days) for $p=0.01,0.02, \ldots 0.05$, with $\beta_{2}=11.65$ corresponding to $q_{2}=0.2$.

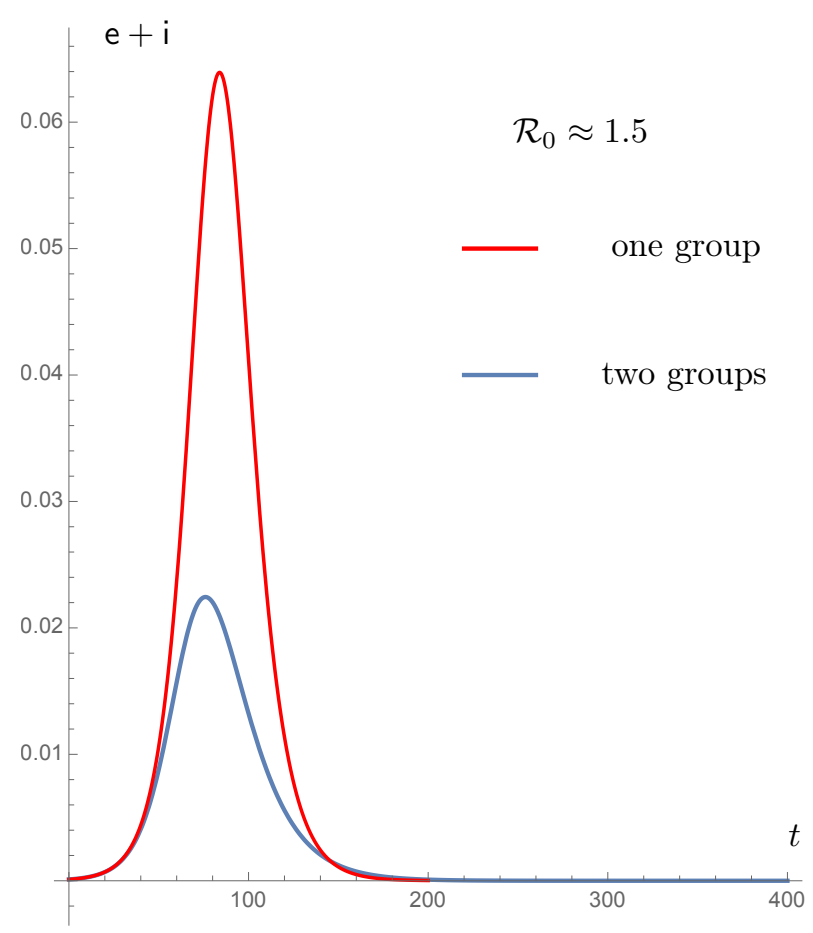

FIGURE 5. A striking effect of the heterogeneity is the flattening of the curve shown here for $\mathcal{R}_{0} \approx 1.5$.

Now, let us describe how the epidemic peak depends on the parameters in our two groups example. For a given value of $\beta_{2}$, larger values of $p$ mean a larger $\mathcal{R}_{0}$, with a linear dependence given by (2), a larger epidemic size (see Fig. 2), but also a larger epidemic peak (see Fig. 6). Let us denote by $t_{\star}$ the date of the peak and let 

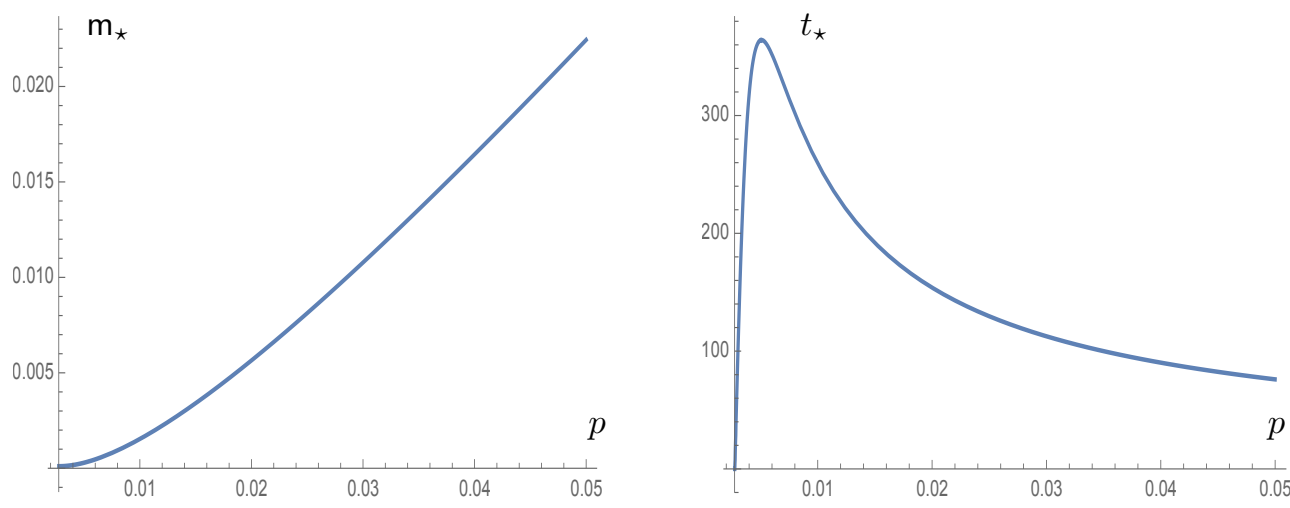

Figure 6. Size (left) and date (right) of the epidemic peak as function of $p$ in the range $0.27 \%$ to $5 \%$. With our choice of the parameters $\left(q_{1}=2.4\right.$ and $\left.q_{2}=0.2\right)$, there is no outbreak if $p \leq 0.27 \%$, because $\mathcal{R}_{0} \leq 1$ in that case.

$\mathrm{m}_{\star}:=\mathrm{e}\left(t_{\star}\right)+\mathrm{i}\left(t_{\star}\right)=\max _{t \geq 0}(\mathrm{e}(t)+\mathrm{i}(t))$. We observe that $p \mapsto \mathrm{m}_{\star}(p)$ is increasing with $p$ and $t_{\star}$ is non-increasing with $\mathcal{R}_{0}$ for sufficiently large values of $\mathcal{R}_{0}$.

It is also interesting to make comparisons with the same value of $\mathcal{R}_{0}$. In our model case for $n=2$, using (2) and the definition of $\beta_{k}$, we can for instance choose $q_{2}$ as a function of $p$ to achieve a fixed value of $\mathcal{R}_{0}$ and get

$$
q_{2}=\frac{\beta p q_{1}}{\gamma q_{1} \mathcal{R}_{0}-(1-\beta) p} .
$$

See Fig. 7 for some qualitative results on the dependence of $\mathrm{m}_{\star}$ and $t_{\star}$ in $\mathcal{R}_{0}$, for various values of $p$. Although not linear, the size of the epidemic peak is a clearly increasing function of $p$ in the outbreak regime. It is an empirical but remarkable fact that, for our set of data, the date of the epidemic peak almost does not depend on $p$.
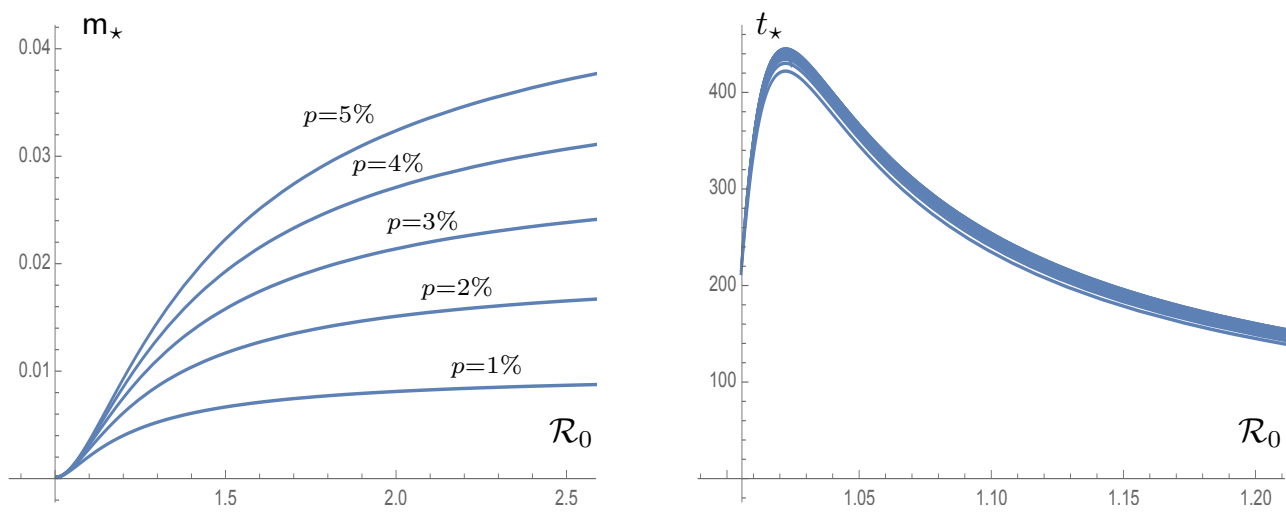

Figure 7. Left: size of the epidemic peak as a function of $\mathcal{R}_{0}$. Right: date of the epidemic peak as a function of $\mathcal{R}_{0}$. Plots correspond to $p=0.01,0.02, \ldots 0.05$. 
The $\mathcal{R}_{0}$ is not the message. As seen in Fig. $7, \mathcal{R}_{0}$ does not retain all relevant information and for a given $q_{1}$ and $\mathcal{R}_{0}$, one may still vary either $p$ or $q_{2}$ and eliminate the other one using (2). Here we shall rely on (5) for a few more plots which show some additional qualitative features of the epidemic size and peak.

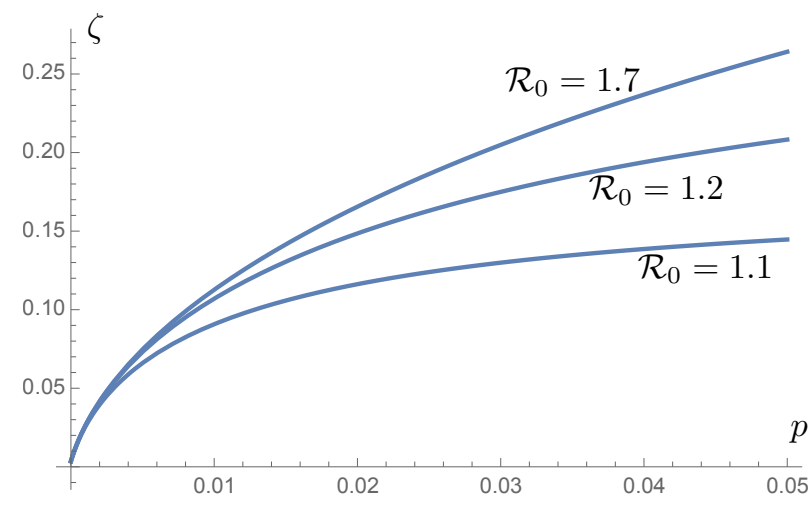

Figure 8. The epidemic size $\zeta$ as a function of $p$ ranging from 0 to 0.05 , where $q_{2}$ is adjusted according to $(5)$ so that $\mathcal{R}_{0}$ is given and equal to 1.1, 1.2 and 1.7.

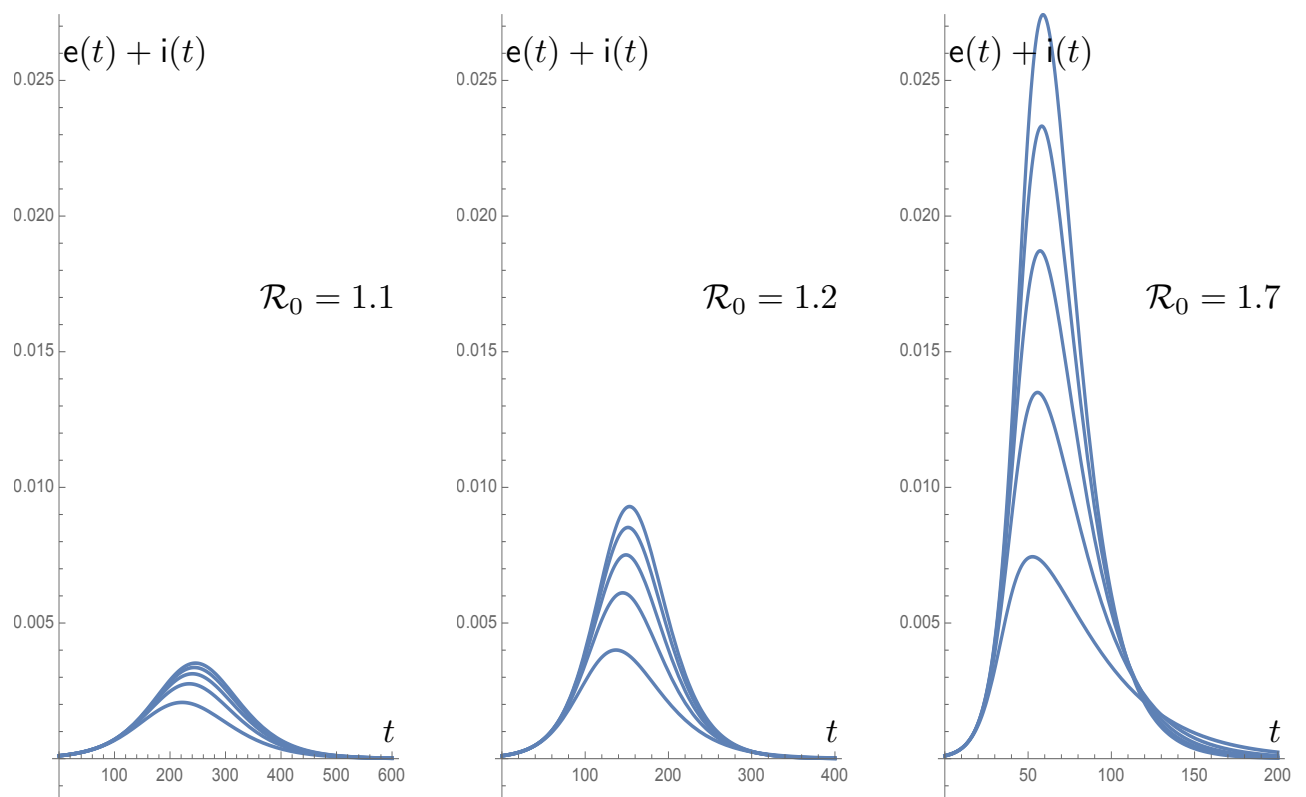

Figure 9. The epidemic curve and the peak strongly depends on $\mathcal{R}_{0}$. Notice that the scale on the vertical axis is the same in the three cases corresponding to $\mathcal{R}_{0}=1.1,1.2$ and 1.7 , while the time scale is respectively $(0,200),(0,400)$, and $(0,600)$. On each plot, the curves correspond to $p=0.01,0.02, \ldots 0.05$. 
Discussion. The goal of a lockdown is to reduce the basic reproduction ratio of the epidemic to a value less than 1 and drive the disease to extinction, or at least decrease it in such a way that "the curve is flattened". The efficiency of the lockdown is achieved by a reduction of social interactions, which is measured (in average) by a factor $q$. In this paper we question whether such an average factor makes sense or not. Beyond the difficult issue of giving realistic values to $q$, we study some consequences of social heterogeneities when the population is divided into groups for which $q$ takes different values.

The basic reproduction ratio $\mathcal{R}_{0}$ has been computed in [4] and is given in (2) as an average of the basic reproduction ratios for each group (or, equivalently, using an average transmission rate) and not as a global ratio based on an average $q$ factor,as it it is implicitly done in many papers in the literature. A small group with a high transmission rate eventually triggers an outbreak even if the basic reproduction ratio of the majority is below 1 . This papers focuses on some quantitative consequences for well chosen numerical examples.

The qualitative properties of the curves in presence of heterogenous groups are not the same as when considering a single group with the same (averaged) basic reproduction ratio. The dynamics of the outbreak and its properties, for instance the height of the epidemic peak er the final epidemic size $\zeta$, are also changed. A model with only one group and fitting the observed data in the initial phase of the outbreak will be more pessimistic concerning the epidemic outcomes than a heterogeneous model. This is even more true after lockdown when social distancing measures have been enforced, the lockdown being by its nature a source of heterogeneity as some individuals are exempted. In terms of public health, this also underlines the importance of targeting prevention measures on individuals with a high level of social interactions. After this study has been completed, important epidemiologic data have been published in [5] which point in the same direction.

Acknowledgment: This work has been partially supported by the Project EFI (ANR-17-CE40-0030) of the French National Research Agency (ANR). The authors thank the MODCOV19 platform [1] for support. The authors thank a referee for very useful comments and especially for pointing an inconsistency due a very inconvenient typo error.

(C) 2020 by the authors. This paper may be reproduced, in its entirety, for non-commercial purposes.

Author's Statement: Authors state no conflict of interest.

\section{REFERENCES}

[1] Modcov19. https://modcov19.math.cnrs.fr.

[2] N. BACAËr, Un modèle mathématique des débuts de l'épidémie de coronavirus en France, Mathematical Modelling of Natural Phenomena, 15 (2020), p. 29.

[3] O. Diekmann, J. A. P. Heesterbeek, and M. G. Roberts, The construction of nextgeneration matrices for compartmental epidemic models, Journal of The Royal Society Interface, 7 (2009), pp. 873-885.

[4] J. Dolbeault and G. Turinici, Heterogeneous social interactions and the COVID-19 lockdown outcome in a multi-group SEIR model, Mathematical Modelling of Natural Phenomena, 15 (2020), p. 36.

[5] EpICov, En mai 2020, 4,5\% de la population en France métropolitaine a développé des anticorps contre le SARS-CoV-2, Etudes \& Résultats, 1167 (2020), pp. 1-6. https://drees.solidarites-sante.gouv.fr/IMG/pdf/er1167.pdf. 
medRxiv preprint doi: https://doi.org/10.1101/2020.05.15.20103010; this version posted January 11, 2021. The copyright holder for this preprint (which was not certified by peer review) is the author/funder, who has granted medRxiv a license to display the preprint in perpetuity.

\section{It is made available under a CC-BY-NC-ND 4.0 International license .}

[6] K. M. A. Kabir And J. TAnimoto, Evolutionary game theory modelling to represent the behavioural dynamics of economic shutdowns and shield immunity in the COVID-19 pandemic, Royal Society Open Science, 7 (2020), p. 201095.

[7] H. Salje, C. T. Kiem, N. Lefrancq, N. Courtejoie, P. Bosetti, J. Paireau, A. Andronico, N. Hozé, J. Richet, C.-L. Dubost, Y. L. Strat, J. Lessler, D. Levy-Bruhl, A. Fontanet, L. Opatowski, P.-Y. Boelle, and S. Cauchemez, Estimating the burden of SARS-CoV-2 in france, Science, 369 (2020), pp. 208-211.

[8] Santé Publique France, French national public health agency. https://dashboard.covid19.data.gouv.fr, 2020. 